\title{
Role of Vibrio tapetis in the development of skin ulceration in common dab (Limanda limanda)
}

Vercauteren Maaike', Annelies Declercq', Evelien De Swaef ${ }^{2}$, Lisa Devriese ${ }^{3}$, Hans Polet ${ }^{4}$, Annemie Decostere' and Koen Chiers'

1 Departement of Pathology, Bacteriology and Poultry Diseases, Faculty of Veterinary Medicine, Ghent University, Salisburylaan 133, 9820 Merelbeke, Belgium E-mail: maaike.vercauteren@ugent.be

2 Departement of Morphology, Faculty of Veterinary Medicine, Ghent University, Salisburylaan 133, 9820 Merelbeke, Belgium

3 Animal Sciences Unit - Aquatic Environment and Quality, Institute for Agricultural and Fisheries Research (ILVO), Ankerstraat 1, 8400 Oostende, Belgium

4 Fishery and Aquatic Production, Institute for Agricultural and Fisheries Research (ILVO), Ankerstraat 1, 8400 Oostende, Belgium

The increasing prevalence of skin ulcers in commercially important flatfish nowadays is an important threat to the fishing industry. Until now, a clear understanding on the possible causes of this disease is still missing. Environmental factors such as temperature rise, fluctuations in salinity, the impact of electric fishing, as well as skin trauma and infections with pathogenic bacteria, such as infection Vibrio tapetis, are suspected to play a role in the development of these skin ulcers. To investigate the impact of these parameters, an infection model whereby skin ulcers can be induced, is developed.

In the current study, individually tagged wild-caught common dab (Limanda limanda) as representatives for marine flatfish were used. Sixty fish were divided in five groups of 12 animals each. To evaluate whether mechanical damage might play a role in ulcer development, from each fish three different areas both on the pigmented and non-pigmented side were treated as follows: removal of mucus, descaling, no damage. The order of the three treatments was randomized for all individuals. Following mechanical treatment, the animals of three groups were challenged by immersion in a suspension of $V$. tapetis $\left(3 \times 10^{5}\right.$ colony forming units $/ \mathrm{ml}$ ), recovered in pure culture from a skin ulcer of a wild-caught dab. Two non-treated control groups were immersed in a tank with water without $V$. tapetis. The fish were clinically monitored for three weeks post-challenge, and morbidity and mortality data recorded. In case humane endpoints were reached, the fish were sacrificed and a full necropsy performed.

Mortality during the experimental period was higher in the challenged group compared to the control group. The fish that died after inoculation displayed dark red ulcerated skin wounds with subcutaneous bleeding at the areas from which the mucus and scales had been removed prior to immersion. Of the animals surviving the experiment, six challenged individuals showed severe ulcerative lesions three weeks post inoculation. In all other surviving animals (challenged and control), variable healing was observed.

Skin (ulcer) samples and samples of internal organs are currently processed for bacteriological, histological and immunohistochemical examination to elucidate the role of Vibrio tapetis in the development of lesions and/or mortality and to decipher the interaction of the pathogen with its host. To conclude, the hitherto obtained results point towards Vibrio tapetis possibly having an etiological role in the development of skin ulcerations in dab subsequent to prior skin damage. However, this research hypothesis needs to be rectified by means of the results of the on-going analyses.

Keywords: skin ulcer; Vibrio tapetis; infection model 